\title{
openheart The HASTE Protocol: a standardised CT Coronary Angiography service operated from a District General Hospital
}

\author{
lain Thomas Parsons, ${ }^{1}$ Clare Bannister, ${ }^{1}$ John Badelek, ${ }^{2}$ Mark Ingram, ${ }^{2}$ \\ Emma Wood, ${ }^{2}$ Alex Horton, ${ }^{2}$ Michael Hickman, ${ }^{1}$ Edward Leatham ${ }^{1}$
}

To cite: Parsons IT, Bannister C, Badelek J, et al. The HASTE Protocol: a standardised CT Coronary Angiography service operated from a District General Hospital. Open Heart 2018;5:e000817. doi:10.1136/ openhrt-2018-000817

Received 7 March 2018 Revised 24 May 2018 Accepted 13 June 2018

Check for updates

(C) Author(s) (or their employer(s)) 2018. Re-use permitted under CC BY-NC. No commercial re-use. See rights and permissions. Published by BMJ.

${ }^{1}$ Department of Cardiology, Royal Surrey County Hospital NHS Foundation Trust, Guildford, UK

${ }^{2}$ Department of Radiology, Royal Surrey County Hospital NHS Foundation Trust, Guildford, UK

Correspondence to Dr lain Thomas Parsons: iainparsons@doctors.org.uk

\section{ABSTRACT}

Introduction CT coronary angiography (CTCA) has excellent sensitivity but lacks specificity when compared with invasive coronary angiography (ICA) particularly in patients with a high coronary calcium burden. CTCA has been shown in large trials to decrease the requirement for diagnostic ICA and provide diagnostic clarity. We describe the methodology used to provide a standardised CTCA service established in a District General Hospital, which may assist other hospitals aiming to develop a cardiac CT service.

Methods Scan request forms, authorisation and patient instruction were recorded. Patient preparation prior to CTCA as well as exclusion and inclusion criteria were documented. Scans were interpreted using a multidisciplinary team (MDT) approach in order to organise follow-up, medication and further investigation.

Results Over 6 months, 157 consecutive scans were performed. CTCA was completed in $88 \%(n=138 / 157)$ and considered of diagnostic quality in $82 \%(n=129 / 157)$. The median radiation dose was $3.42 \mathrm{mSv}$. Overall, $64 \%$ of patients had evidence of coronary calcium. Following MDT review, $72 \%$ ( $n=113 / 157)$ of patients were discharged without requiring invasive angiography. $15 \%(n=24 / 157)$ of patients went on to have invasive angiography showing non-obstructive disease and $13 \%$ (20/157) of patients underwent percutaneous coronary intervention $(11 \%)$ or bypass surgery (1\%).

Discussion Appropriate referrals, patient preparation and scan quality remain significant factors in running a CTCA service. Despite this, the vast majority of patients can be discharged on the basis of the CTCA alone. An MDT approach is key to the delivery of a cardiac CT service.

\section{INTRODUCTION}

Atherosclerotic coronary artery disease (CAD) remains a leading cause of death in the UK. ${ }^{1}$ Chest pain is a frequent reason for referral for invasive coronary angiography (ICA). While ICA is the gold standard for the diagnosis of CAD, it is a resource- intensive investigation associated with a small risk of serious complication and in many hospitals often confirms normal or non-obstructed coronary arteries. ${ }^{2}$ CT coronary angiography (CTCA) has proved to be a safe and non-invasive investigation to diagnose and

\section{Key questions}

What is already known about this subject?

- CT coronary angiography is the non-invasive test of choice for the investigation of stable coronary artery disease.

- A considerable upscale in the provision of CT coronary angiography is required

What does this study add?

- A detailed description of the methodology for the provision of a cardiac CT service from a District General Hospital.

- Overall, $72 \%$ of patients can be discharged without the requirement for invasive angiography.

- There remains a requirement for the provision of functional testing.

How might this impact on clinical practice?

- The methodology may serve as a standard that may assist other District General Hospitals in developing a diagnostic cardiac CT service.

- A close working relationship between cardiology and radiology is key to the delivery of an effective service.

risk-stratify patients with CAD. CTCA has diagnostic accuracy comparable with $\mathrm{ICA}^{3-5}$ with excellent sensitivity but can lack specificity particularly in patients with a high coronary calcium score. The tendency to overestimate stenotic plaque disease and so inaccurately define flow-limiting disease remains a challenge and has led to concerns about the effectiveness of CTCA due to the incidence of false-positive results. Large trials have shown that availability of CTCA decreases the incidence of non-obstructive CAD at ICA while increasing the overall number of ICA. $^{6-10}$ Additionally, several prognostic features have been investigated with CTCA including the calcium burden, total plaque burden as well as the presence of high-risk plaques, which have been shown to have prognostic value in predicting death or non-fatal myocardial infarction. ${ }^{11-13}$ CTCA can also diagnose 
non-obstructive atherosclerotic plaque disease, which permits risk factor modification in high-risk cases potentially years before coronary ischaemia occurs. For all of these reasons, we established a cardiac CT (CCT) service in our District Hospital.

The 2016 National Institute for Health and Care Excellence (NICE) guidance has put CTCA at the forefront of initial investigation for stable CAD in the UK. ${ }^{14} 15$ Currently, however, the UK provision of CTCA outside of high-volume centres is patchy. ${ }^{14}$ It is estimated that a $700 \%$ increase in the availability of CTCA is required across the country, a considerable upscale in the provision of the service. ${ }^{14}$ It remains to be seen whether the accuracy of recent large CTCA trials ${ }^{7}$ can be reproduced in smaller centres using often less advanced technology. The aim of this paper was to describe the methods and results of a District General Hospital (DGH), both successes and pitfalls, which may potentially be used as a model for the introduction or development of a CCT service elsewhere.

\section{METHODS}

We based our methods on the British Society of Cardiac Imaging Standards of Practice of CT Coronary Angiography in adult patients and Society of Cardiac Computed Tomography guidelines for the performance and acquisition of coronary CT angiography. ${ }^{16} 17$ These guidelines remain the 'gold standard', and our paper shows how we have attempted to deliver a high-quality imaging practice (with sound selection of patients, patient preparation, CT acquisition, post-processing methods and onward care) but without a consultant cardiologist or radiologist dedicated to cardiac CT and basic scanner and post-processing technology. An overview of our pathway is in figure 1 .

\section{REFERRAL SCREENING}

Requests for CCT scans in our institution require verification by a consultant cardiologist to check appropriateness. The scans were predominantly requested by one of our five consultant cardiologists, their specialist trainees or chest pain assessment nurse specialists. Referrals were from ward inpatients, the nurse-led rapid access chest pain clinic (RACPC) or from cardiology outpatient clinic. All referrals required completion of a cardiology imaging request form (viewable at http:/ / haste.uk.com/ scan-appeal/forms/). The referral request form required the referrer to stipulate any contraindications to betablockade, renal function, presence of atrial fibrillation, pregnancy, pacemaker presence, left bundle branch block or metformin. In our hospital, one request form is used for all our specialist cardiac imaging modalities: dobutamine stress echocardiography, cardiovascular magnetic resonance, myocardial perfusion scintigraphy and CTCA. Authorisation for all investigations by a consultant cardiologist minimised unsuitable referrals and simplified redirection of inappropriate imaging requests to more suitable cardiac imaging modalities.

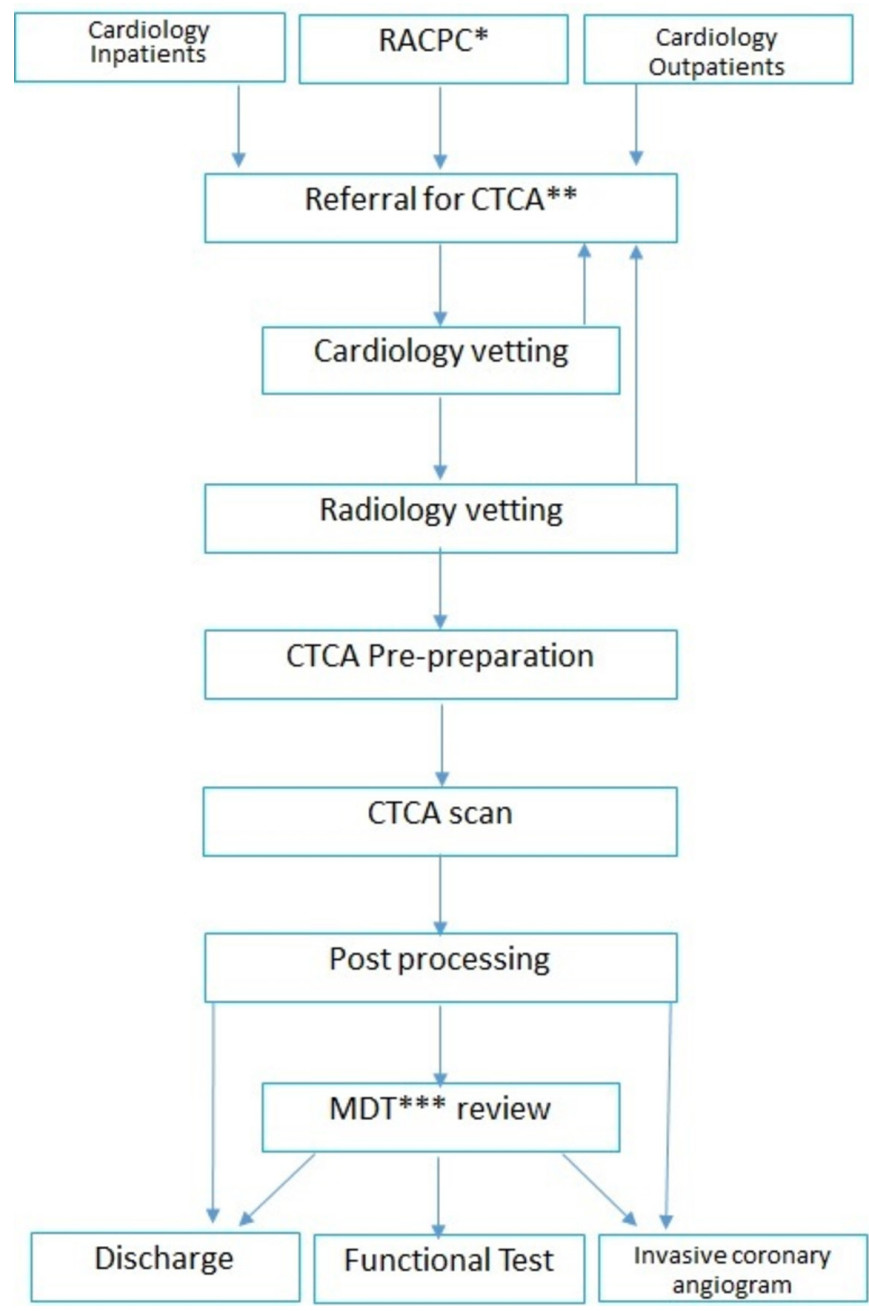

Figure 1 Heart and Stroke Trust Endeavour (HASTE) pathway. ${ }^{\star}$ Rapid access chest pain clinic, ${ }^{* *} \mathrm{CT}$ coronary angiogram, ${ }^{\star \star \star}$ multidisciplinary team meeting.

The aim of referral screening was to maximise diagnostic yield from all cardiac investigations and minimise the number of inappropriate, poor-quality or abandoned CCT scans. Following cardiologist vetting, a radiologist sanctioned referrals to comply with ionising radiation (medical exposure) regulations. Consultant radiologists also provided onsite supervision of all scans in case of additional prescription requirements.

All patients were provided with clear written instructions on what to expect including the requirement to be collected following the scan. This advice followed some early incidents where patients had unusually low heart rates or hypotension following beta-blocker/glyceryl trinitrate (GTN) administration making unsupervised travel or driving ill-advised. This also allowed the option of sedation to be administered where anxiety-driven tachycardia was unresponsive to standard beta-blockers. All patients were asked to take any prescription medications on schedule as normal (including beta-blockers or calcium channel blockers), refrain for eating for 4 hours prior to the scan and to avoid caffeinated beverages and other stimulants for 6 hours prior. Patients were often, 
but not routinely, pre-started on oral beta-blockers prior to the scan, which served as an anti-angina agent as well as assisted in heart rate control.

\section{CTCA PRE-CHECK PROCEDURE}

All patients were checked in by a qualified nurse and had basic observations recorded including pulse rate and blood pressure. Soon after arrival in the department, patients were asked to complete a comprehensive safety questionnaire based on Royal College of Radiologists recommendations. ${ }^{17}$ Patients were excluded from the CTCA, although captured for the purposes of this Registry, if found to have poorly controlled asthma on daily bronchodilators or atrial fibrillation/flutter with a ventricular rate exceeding 60 beats per minute. Other exclusion criteria were prior cardiac CT imaging that showed heavy cardiac calcification, prior coronary stents or the patient's body mass index exceeded $35 \mathrm{~kg} / \mathrm{m}^{2}$.

Nursing staff or radiographers placed a minimum 20G peripheral venous cannula for the purposes of administering intravenous contrast. All cannulas were tested prior to contrast delivery with a rapid infusion of intravenous $0.9 \%$ saline. Wherever possible, a right-arm cannula was inserted to prevent streak artefact from undiluted contrast in the left brachiocephalic vein where it crosses the midline. The patient's heart rate was rechecked $30 \mathrm{~min}$ after the initial administration of oral beta-blocker, and if it still exceeded $60 \mathrm{bpm}$, further oral metoprolol or oral/ intravenous lorazepam was then prescribed at the discretion of the supervising radiologist. Other negatively chronotropic medications such as ivrabradine or diltiazem were not used unless they already formed part of the patient's prescription. Every effort was made to ensure every patient was as relaxed as possible prior to the scan.

On achieving satisfactory heart rate control, the patient was positioned on the gantry table. Three ECG electrodes were placed in the left and right third intercostal space midclavicular line as well as the ninth intercostal space midaxillary line (modified Einthoven's triangle). ${ }^{18}$ The aim was to allow for the best possible ECG signal (so allowing for the best recognition of ECG trigger points) but without unnecessary impingement on the field of view resulting in streak artefact or image noise. Where the patient's blood pressure exceeded 90/60 $\mathrm{mm} \mathrm{Hg}, 400$ $\mu \mathrm{g}$ of GTN was given sublingually to dilatate the coronary arteries. This allowed for time for transient increase in heart rate to settle; a frequent consequence of sublingual GTN administration.

Commonly experienced sensations to contrast were explained to the patients to help prevent an increase in heart rate during the contrast phase. Breath-hold techniques were practised prior to image acquisition. This allowed the radiographer to determine the delay from asking the patients to breathe in to initiating the scan. This is typically $6 \mathrm{~s}$ (range $1-7 \mathrm{~s}$ ).

At any point up to the main contrast injection, wherever the heart rate control was deemed inadequate
( $<65 \mathrm{bpm})$, the scan process was halted. At the discretion of the radiologist, the scan was either abandoned, a calcium score only was obtained or wherever logistically possible the patient was taken off the table and prescribed more beta-blockade \pm lorazepam before a further attempt later in the same session-typically 30-60 min later. All patients had a calcium score obtained first. While many centres will abandon the scan where there was heavy calcium deposition and use the Ca score as a scout, we had no predefined cut-off and scans were not abandoned secondary to heavy coronary calcification. The radiographer used the first scan to check the field of view and position the first (cephalic end) slice of the contrast scan just above the origin of the left main coronary artery to minimise the chance of step artefact involving the proximal part of the coronary tree (left main stem (LMS) or proximal left anterior descending coronary artery $(\mathrm{LAD}))$.

\section{CTCA ACQUISITION}

Most scans were performed on a GE LightSpeed VCT XTE 64 slice CT Scanner (GE Healthcare, Chicago, Illinois, USA); however, a small proportion were performed on a Siemens Somatom Definition 128-slice CT scanner (Siemens Medical Solutions, Erlangen, Germany). Scans were performed by experienced cardiac CT-trained radiographers irrespective of scanner. Each CTCA list was supervised by a consultant radiologist with a minimum of Level 2 accreditation in CCT.

For contrast, lopamidol, $120 \mathrm{~mL}$ ( $370 \mathrm{mg} / \mathrm{mL}$ ) (Niopam 370; Bracco S.p.A., Milan, Italy), was used with $0.9 \%$ sodium chloride bolus chaser. Initially, a $20 \mathrm{~mL}$ test bolus of contrast with a $20 \mathrm{~mL}$ saline push was given using the Multiple Image Region of Interest (MIROI) technique to time the contrast in the coronary arteries. Multiple scans are taken at the same anatomical location. The measurement scale used was the Hounsfield unit (HU) in relative or absolute scale. The relative scale displays the first reading as zero and the difference in CT numbers from one scan to the next. The absolute scale displays the actual HU number and the difference from one scan to the next. The region of interest (in this case the aorta) is selected using an elliptical, box or trace function in the location to be measured. MIROI function gives a graphic of CT number change over time. Around $9 \mathrm{~s}$ is typically added to decrease superior vena cava (SVC) artefact and to allow contrast to empty from the right ventricle. When the appropriate time has been selected, the remaining $100 \mathrm{~mL}$ of Niopam is given with a further $40 \mathrm{~mL}$ chaser bolus of $0.9 \%$ sodium chloride to maximise contrast dose and to minimise streak artefact in the SVC. The typical time from contrast to acquisition is $27 \mathrm{~s}$. In graft studies, higher doses of contrast were used as the scan is required to start from the level of internal mammary artery origin. We did not routinely use contrast minimising techniques. Where issues with heart rate control were anticipated, prospective padding was applied (typically $30 \%-70 \%$ of 
the RR interval). We did not routinely acquire images only in end-systole. Retrospective gating was used extremely rarely and only with agreement of the supervising radiologist. The tube voltage was not routinely reduced for smaller patients nor increased for larger patients.

Immediately following the scan, patients were warned that they may experience light-headedness and a member of staff was present to assist the patient where required. Patients spent $15 \mathrm{~min}$ in the department following the scan to ensure no adverse effects from beta-blockade, nitrates or contrast.

\section{POST-CTCA}

All CTCAs were post-processed and primarily reported by a consultant radiologist using a dedicated GE Workstation (GE Healthcare, Chicago, Illinois, USA) to calculate a calcium score (Agatston score ${ }^{19}$ ) and perform vessel reconstructions. Reports for normal scans, with low or normal calcium scores, were issued directly to the referring team. Abnormal scans were flagged in preparation for case discussion at a multidisciplinary team (MDT) meeting.

The MDT group comprised a nurse specialist (rapid access chest pain nurse specialist), two consultant cardiologists with subspecialist interest in interventional cardiology and cardiovascular imaging, respectively, and three consultant radiologists with accreditation in CCT. For abnormal scans, a minimum of one cardiologist and one radiologist was required for each report. The MDT, for the purposes of reporting, had access to the original request form, CTCA pathway (containing information about the blood pressure, heart rate and any medication given during the examination) and recent clinic letters.

Reporting was generally performed using clear definitions; for the main coronary vessels, a critical stenosis (CS) was defined as an unequivocal stenosis with reduction in luminal diameter by at least $75 \%$ (including likely occlusion). A possible flow-limiting lesion (PFL) was defined as narrowing of a discrete part of the coronary vessel lumen where there was a coronary stenosis of at least greater or equal to $50 \%$, but no clear occlusion or critical narrowing could be identified. Non-flow limiting (NFL) described minor luminal plaque disease $(<50 \%)$. A comment was also included on every report regarding the 'quality' of the scan. 'Excellent quality' meant all coronary arteries and branches were clearly visualised with no artefacts. 'Reasonable quality' conferred that the proximal vessels were visualised but one or more distal arteries were difficult to fully assess whether due to artefact or poor contrast opacification. 'Poor quality scans' were where one or more proximal main arteries could not be adequately assessed. We found it useful to have a clear reporting strategy to convey information rapidly and accurately among the MDT. Consistency in reporting, in this fashion, was also anecdotally useful for requesting physicians. Each CTCA report comprised a radiologist (predominantly descriptive) report describing the CT calcium score, anatomical arrangement and presence of any CS or PFL, which was later combined with an MDT addendum describing the clinical outcome/conclusion of the CTCA findings along with recommended steps for ongoing care. In the majority of cases, in order to avoid administrative delay, the cardiologist would action these immediately, for example, by dictating a letter or sending email particularly where urgent clinically relevant disease was identified and onward referral for ICA was recommended. High-risk anatomy was generally deemed as a CS in the proximal LAD or LMS or significant widespread disease. Having the nurse specialists who staff the RACPC present in the MDT we found of great value in providing more clinical data on the patients. Interpreting the scan in the clinical context added a further layer of granularity particularly in reasonable or poor scans. Alternate outcomes included referral for functional tests or, in the case of NFL disease, a summary letter to the general practitioner (and patient) with recommended primary prevention. While we did not analyse individual clinician's onward investigations in the presence of PFLs, there was variation dependent on personal preference and test availability.

\section{RESULTS}

Between August 2015 and February 2016, 162 CTCAs were requested. Of these, five (3\%) were excluded from the analysis; two of the patients did not attend their scan and one in whom the scan performed was not a CTCA but a CT aortogram. In a further two patients, the scan request was duplicated so the CTCA was not performed and the patient did not attend the appointment. The characteristics of all patients referred for CCT are detailed in table 1 .

The most common indication for CCTA was to investigate potential stable $\mathrm{CAD}$ accounting for $66 \%$ $(n=103 / 157)$ of requests. In $17 \%$ of requests, the CTCA was requested for the assessment of low-risk acute chest pain. In $5 \%$ of requests, the patient was asymptomatic but the CTCA was performed due to risk factors. In the remaining 12\%, the CTCA was requested for other reasons. This included ruling out CAD prior to cardiothoracic surgery or in the presence of a significant burden of dysrhythmias; commonly ventricular ectopy. In one case, the CTCA was performed because the ICA was non-diagnostic due to inability to catheterise the right coronary artery. Of the 25 inpatients, nine $(36 \%)$ underwent invasive angiography following CTCA with five undergoing eventual percutaneous coronary intervention (PCI). In contrast, $25 \%$ of outpatients underwent eventual invasive angiography.

Overall, both a calcium score and CTCA were completed in $88 \%(n=138 / 157)$ of patients. In $8 \%(n=12 / 157)$ of patients, only a calcium score was completed most commonly due to an inability to satisfactorily control the patient's heart rate. In two patients, this was due to being unable to administer beta-blockade due to poorly 
Table 1 Patient demographics, referral source, preparatory medication and prior tests

\begin{tabular}{|lcl}
\hline Demographics & & \\
\hline Age (mean, SD) & 58 & \pm 12 \\
\hline Men (n, \%) & 99 & $(63 \%)$ \\
\hline Women (n, \%) & 58 & $(37 \%)$ \\
\hline Referral source & $(\mathrm{n})$ & $(\%)$ \\
\hline Cardiology outpatients & 70 & $(45 \%)$ \\
\hline Rapid access chest pain clinic & 61 & $(39 \%)$ \\
\hline Cardiology inpatients & 25 & $(16 \%)$ \\
\hline Cardiothoracic outpatients & 1 & $(1 \%)$ \\
\hline Preparatory medication & & \\
\hline No medication & 39 & $(25 \%)$ \\
\hline Oral metoprolol 50 mg & 10 & $(6 \%)$ \\
\hline Oral metoprolol 100 mg & 43 & $(27 \%)$ \\
\hline Oral metoprolol 150 mg & 38 & $(24 \%)$ \\
\hline Sublingual GTN & 110 & $(70 \%)$ \\
\hline Intravenous lorazepam 0.5 mg & 3 & $(2 \%)$ \\
\hline Prior tests & & \\
\hline None & 5 & $(3 \%)$ \\
\hline Exercise test & 74 & $(47 \%)$ \\
\hline Dobutamine stress echo & 70 & $(45 \%)$ \\
\hline Invasive angiography & 4 & $(3 \%)$ \\
\hline Other & 4 & $(3 \%)$ \\
\hline
\end{tabular}

GTN, glyceryl trinitrate.

controlled asthma. In one patient, there was a reaction to the contrast. In $3 \%(n=5 / 157)$ of cases, the CTCA was abandoned completely with neither a CTCA nor CACS obtained due to poor heart rate control. In the remaining $1 \%(n=2 / 157)$ of patients, a calcium score was not calculated (but was obtained) due to irrelevance to the clinical question. In four patients with a calcium score of $>400$ (930-3251 AU), the CTCA was abandoned.

The overall median radiation dose length product (DLP) overall was 244 (IQR 192-260) mGy-cm (3.42 mSv using a thoracic conversion factor of 0.014 ) with 31 (IQR 25-35) mGy-cm $(0.4 \mathrm{mSv})$ for the CACS-only group and 253 (IQR 195-261) mGy-cm (3.54 mSv) for the CACS and CTCA group. One patient underwent retrospective acquisition with a DLP of $790 \mathrm{mGy}-\mathrm{cm}$. Seven patients required repeat imaging due to contrast timing or movement artefact.

Overall, a quarter $(n=39 / 157)$ of patients did not require metoprolol as they had either endogenously low resting heart rates or were already medicated (table 1). Sublingual GTN was not administered in 19 patients $(n=19 / 157,12 \%)$ due to hypotension or being on phosphodiesterase 5 inhibitors. In $18 \%$ of patients, the administration of GTN was not recorded $(n=28 / 157)$ and therefore was considered not to have been given. When GTN was given, $90 \%$ of scans were of excellent or reasonable quality with $10 \%$ being poor. When GTN was not given (or not recorded to have been given), $62 \%$ of scans were of excellent or reasonable quality with $38 \%$ poor quality $\left(\mathrm{p}<0.001 ; \chi^{2}\right.$ test $)$. Lorazepam sedation was required in three patients for anxiety largely due to claustrophobia. In all cases, only $0.5 \mathrm{mg}$ of intravenous lorazepam was given under the direction of the supervising radiologist.

In $81 \%$ of patients $(n=127 / 157)$, the scan quality was diagnostic. The scan quality was deemed excellent by the reporting radiologist in $62 \%(n=98 / 157)$ of patients. In $19 \%(n=30 / 157)$, the scan quality was deemed reasonable and diagnostic, defined as visualisation of all coronary arteries and diagnostic proximal vessels. In $19 \%$ $(n=29 / 157)$, the scan was not diagnostic (table 2$)$. This included where the scan was performed but of too poor quality to be diagnostic $(n=11,7 \%)$, the CT coronary angiography was not performed and only a CACS was acquired $(n=13,8 \%)$ or the scan was abandoned $(n=5$, $3 \%)$. There was a contrast reaction in one patient who did not require hospitalisation. There were no recorded cases of contrast extravasation.

The median calcium score was 21 (IQR 0-228). In 36\% $(\mathrm{n}=57 / 157)$ of patients, the calcium score was 0 . Overall, $15 \%(n=23 / 157)$ of patients had a Ca score of $>400 \mathrm{AU}$ and 19 of these patients were sent for angiography at MDT of which nine underwent subsequent PCI. Following the CTCA, 31\% ( $n=49 / 157)$ of patients had no evidence of CAD with a further $24 \%(n=38 / 157)$ having NFL disease. In 29\% $(\mathrm{n}=46 / 157)$, there was calcium and PFL disease or a CS on CTCA with a further $3 \%(n=5)$ having non-calcified PFL disease or a CS. In the remaining patients $(\mathrm{n}=19)$, the CTCA was not performed as described above.

The overall outcome was that $54 \%(n=85 / 157)$ of patients were able to be discharged from cardiology (table 3). On the basis of the CTCA and MDT decision, $8 \%(13 / 157)$ of patients were referred directly for PCI (all completed). In a further 3\% (5/157), a diagnostic ICA was performed prior to referral for PCI. The median follow-up time was 59 days (range 0-394 days). In the follow-up period, one patient who was discharged with primary prevention underwent an ICA, which confirmed no obstructive disease. No patients who were discharged on the basis of a normal CTCA had an ICA. No patients who were sent for ICA by the MDT did not complete it. There were no deaths in the follow-up period.

\section{DISCUSSION}

American College of Cardiology registry data have previously shown that in North America, 38\% of patients, during ICA, have obstructive CAD at ICA (defined as stenosis $>70 \%$ ) with no CAD seen in $39 \%$ of patients. ${ }^{2}$ In our catheter laboratory experience, an even higher proportion of ICAs have no need for revascularisation. With our CTCA protocol, following MDT review, 72\% $(n=113 / 157)$ were discharged without requiring an 
Table 2 Quality of the scan impact on the multidisciplinary team outcome and further investigative management

\begin{tabular}{|c|c|c|c|c|c|c|}
\hline \multirow{2}{*}{ Multidisciplinary team outcome } & \multicolumn{2}{|c|}{ Excellent quality } & \multicolumn{2}{|c|}{ Reasonable quality } & \multicolumn{2}{|c|}{ Poor quality } \\
\hline & & & & & & \\
\hline ICA \pm proceed & 22 & $(22 \%)$ & 5 & $(17 \%)$ & 1 & $(3 \%)$ \\
\hline ICA only & 2 & $(2 \%)$ & 2 & $(7 \%)$ & 5 & $(17 \%)$ \\
\hline Discharged with no prevention & 24 & $(24 \%)$ & 6 & $(20 \%)$ & 2 & $(7 \%)$ \\
\hline Discharged with prevention & 42 & $(43 \%)$ & 10 & $(33 \%)$ & 1 & $(3 \%)$ \\
\hline Functional test: MPS/DSE & 5 & $(5 \%)$ & 6 & $(20 \%)$ & 9 & $(31 \%)$ \\
\hline Other & 3 & $(3 \%)$ & 1 & $(3 \%)$ & 11 & $(38 \%)$ \\
\hline \multicolumn{7}{|l|}{ Management outcome } \\
\hline ICA: non-obstructive disease & 16 & $(16 \%)$ & 4 & $(13 \%)$ & 4 & $(14 \%)$ \\
\hline ICA proceeded $\mathrm{PCI}$ & 9 & $(9 \%)$ & 3 & $(10 \%)$ & 1 & $(3 \%)$ \\
\hline ICA referred for $\mathrm{PCl}$ & 2 & $(2 \%)$ & 1 & $(3 \%)$ & 2 & $(7 \%)$ \\
\hline No ICA performed & 69 & (70\%) & 22 & $(73 \%)$ & 22 & $(76 \%)$ \\
\hline Surgical management & 2 & $(2 \%)$ & 0 & $(0 \%)$ & 0 & $(0 \%)$ \\
\hline Total & 98 & (62\%) & 30 & (19\%) & 29 & (18\%) \\
\hline
\end{tabular}

DSE, dobutamine stress echocardiogram; ICA, invasive coronary angiogram; MPS, myocardial perfusion scan; PCl, percutaneous coronary intervention.

ICA, although of these, 17 patients $(15 \%, \mathrm{n}=17 / 113)$ also had a functional test.

Overall, $27 \%(\mathrm{n}=42 / 157)$ of patients had an ICA of which in $57 \%(\mathrm{n}=24 / 42)$ of patients the ICA confirmed non-obstructive disease $(\mathrm{n}=4$ requiring pressure wire and fractional flow reserve). The remaining $43 \%(n=18 / 42)$ underwent PCI with 13 of these performed immediately and the remaining five performed at a later date. A CTCA with MDT-guided approach may decrease the number of ICA showing non-obstructive disease when compared with a functional test-based approach. ${ }^{6}$ While our referral rates for ICA were greater than the $12 \%$ seen in the
SCOT-HEART study, our data set also included patients with low-risk troponin-positive chest pain admitted as inpatients rather than the patients with stable angina seen exclusively in SCOT-HEART. Regardless, $25 \%$ of our outpatients underwent invasive angiography, and this is likely due to poor image quality. In comparison, the majority of our scans were performed on 64-detector-row CT scanners rather than the 320-detector-row scanners in the SCOT-HEART trial. The more judicious use of functional tests may improve the yield of PCI, which was reflected also in the 2016 NICE guidance update. ${ }^{15}$ Recent developments in the calculation of fractional flow

Table 3 Multidisciplinary team outcome versus invasive coronary angiography

\begin{tabular}{|c|c|c|c|c|c|c|c|}
\hline \multirow[b]{2}{*}{ Multidisciplinary team outcome } & & & \multicolumn{5}{|c|}{ Invasive coronary angiogram (ICA) outcome } \\
\hline & & & No ICA & $\begin{array}{l}\text { ICA: no } \\
\text { obstructive } \\
\text { disease }\end{array}$ & $\begin{array}{l}\text { ICA refer } \\
\text { for } \mathrm{PCl}^{*}\end{array}$ & $\begin{array}{l}\text { ICA, FFR† } \\
\text { and PCI }\end{array}$ & $\begin{array}{l}\text { ICA for } \\
\text { CABG‡ }\end{array}$ \\
\hline Discharged from cardiology & 85 & $(55 \%)$ & & & & & \\
\hline Discharged with primary prevention & 35 & $(22 \%)$ & 34 & 1 & 0 & 0 & 0 \\
\hline Discharged with reassurance & 50 & $(32 \%)$ & 50 & 0 & 0 & 0 & 0 \\
\hline Referred for functional test & 20 & $(13 \%)$ & & & & & \\
\hline Dobutamine stress echo & 14 & $(9 \%)$ & 17 & 2 & 1 & 0 & 0 \\
\hline Myocardial perfusion scintigraphy & 6 & $(4 \%)$ & & & & & \\
\hline Referred for angiography & 37 & $(24 \%)$ & & & & & \\
\hline Invasive angiography only & 9 & $(6 \%)$ & 0 & 5 & 3 & 1 & 0 \\
\hline Invasive angiography \pm proceed & 28 & $(18 \%)$ & 0 & 15 & 1 & 12 & 0 \\
\hline Other & 15 & $(10 \%)$ & 12 & 1 & 0 & 0 & 2 \\
\hline Total & & & $113(72 \%)$ & $24(15 \%)$ & $5(3 \%)$ & $13(8 \%)$ & $2(1 \%)$ \\
\hline
\end{tabular}

CABG, coronary artery bypass grafting; ICA, invasive coronary angiogram; FFR, fractional flow reserve; PCI, percutaneous coronary intervention. 
reserve non-invasively (FFRCT) have been highlighted in three large multicentre studies (NXT, DISCOVER-FLOW and DeFACTO) comparing FFRCT with invasive FFR measurements. ${ }^{20-22}$ The high negative predictive value of FFRCT may also improve the yield of PCI. Recently, the Prospective Longitudinal Trial of FFRCT: Outcome and Resource Impacts (PLATFORM) study has investigated the clinical use of FFRCT, and the results revealed that CCTA with FFRCT did in fact lead to a marked reduction in the number of ICA showing no obstructive CAD. ${ }^{7}$ This was a non-randomised study and there was no comparison with CTCA alone, and further study is therefore warranted.

The main aim of our protocol was to maximise the number of diagnostic scans. We aimed to use cardiologist's knowledge of the strengths and weaknesses of CTCA, although training in the modality remains variable. Although we did not assess what percentage of referrals were diverted to alternate imaging modalities, we were able to complete $89 \%(n=140 / 157)$ of examinations with $82 \%$ ( $n=129 / 157)$ being of excellent or reasonable quality in providing diagnostic information. Unfortunately, $19 \%(29 / 157)$ of our scans were non-diagnostic in that they were unable to answer the clinical question. However, in 5 patients, the scan was abandoned due to inability to control the heart rate, and in 11 patients, the scan was performed but of too poor quality. In the remainder, a calcium score was obtained only giving prognostic information, but further tests were required.

This demonstrates the paramount importance of pre-CTCA patient selection and preparation particularly when using less modern scanner technology.

Early in our experience, we found oral metoprolol $50-150 \mathrm{mg}$ to be simpler and more effective in consistently lowering the heart rate compared with intravenous bolus metoprolol 10-20 mg. We adopted a patient group directive method for administration of oral metoprolol pre-CT. We acknowledge that oral metoprolol requires an area where the patients can wait for the medication to take appropriate effect, and this may not be available in many hospitals. As a service, we have been through periods of using and not using sublingual GTN to increase coronary artery diameter, hence GTN was only used in $70 \%$ of patients. The use of GTN is, however, well established in terms of improving coronary artery visualisation and is recommended in guidelines. ${ }^{16}{ }^{20}$ Retrospectively, we have found a statistically significant subjective improvement in scan quality when GTN was given.

Regardless of the number of CTCA requests diverted to other imaging modalities, there will always be the requirement for other functional imaging modalities. The main reason for an abandoned or poor-quality, non-diagnostic scan was due to poor heart rate control. The SCOTHEART trial quoted that they obtained 'diagnostic information in 99\% of patients' despite the inclusion of obese patients with high calcium scores and atrial fibrillation. However, the SCOT-HEART trial excluded patients over 75 years and, in the CTCA arm, had non-compliance in
$14 \%(\mathrm{n}=295 / 2073)$. In our cohort, the combination of consultant cardiologists vetting requests with directed scanning protocols is comparable with larger trials despite having no upper age cap. With increasing scanner technology, which often is not available in DGHs, the capability to obtain diagnostic images at higher heart rates will mitigate this further.

The radiation dose of diagnostic ICA is reported to be around $7 \mathrm{mSv}$ (range $2-16 \mathrm{mSv}$ ). ${ }^{21}$ By comparison, newer dose reduction strategies and prospective triggered CTCA scanning have resulted in a radiation dose decrease $^{22}$ from $21.1 \mathrm{mSv}$ to $4.3 \mathrm{mSv}$ with no significant reduction in diagnostic performance. ${ }^{23}$ Studies can now be acquired with $<1 \mathrm{mSv}$ of radiation, although heart rate control remains crucial. ${ }^{7}$ In the SCOT-HEART trial, the median radiation dose was 4.1 (3.0-5.6) mSv (DLP 291), with greater than one-third of this dose attributable to the measurement of the coronary calcium score. ${ }^{7}$ Our median dose, using the same thoracic conversion factor, was $3.54 \mathrm{mSv}$ in patients who completed both the CTCA and CACS. Whether the thoracic conversion factor of 0.014 is acceptable for mediastinal imaging is debatable, but even in terms of DLP, we are comparable with the national median dose of $209 \mathrm{mGy}-\mathrm{cm}^{24}$ This could be reduced further with tube voltage modulation, improved scanner technology ${ }^{2526}$ and calculating the calcium score from the CTCA images ${ }^{27}$ or, as per the 2016 NICE guidance update, ${ }^{15}$ not performing a prior calcium score.

Our hospital is not a PCI centre, so part of the CTCA pathway is to improve efficiency of onward referrals for ICA. Where a diagnostic angiogram was deemed necessary for exclusion of significant disease but with a low probability of requiring intervention, the patient was listed for invasive angiogram in our own hospital without an option for PCI. We feel that this approach improves efficiency and helps reduce the number of patients referred to other Trusts unnecessarily.

The MDT remains an essential part of our protocol to assess, on a case-by-case basis, which PFL or CS lesions would be amenable or inappropriate for intervention. We found the collaboration between cardiologist and radiologist to be of paramount importance in the delivery of our service to ensure rapid senior decision-making and the avoidance of completed scans with potentially critical disease going un-actioned. The onward management of patients at the time of MDT further decreases delays. Adding an MDT addendum to the CTCA report serves as a further record for MDT discussion.

There are several limitations to our study. We thought it useful to illustrate the outcome differences between the excellent, reasonable and poor-quality scans and so have divided our results as such to provide as much detail as possible. However, there are no national or local guidelines to assess the quality of the CTCA scan. The scan quality is therefore without clear definition and down to the interpretation of the individual radiologist, which is open to bias. Routine collection of the heart rate at the time of scanning would be a useful measure to audit 
pre-scan preparation. This is a single-centre, non-randomised, retrospectively analysed prospective cohort survey, which is therefore susceptible to the usual biases. To minimise bias, our data analysis was performed by trainee cardiologists who did not participate in the MDT and who had not requested any of the scans (hence the period of sampling). This study was not powered to assess for major adverse cardiac events nor was there a control group that accounts for the limited follow-up. A further retrospective analysis of these data may prove informative with regard to clinical outcomes, although this has been done in larger trials. ${ }^{13}$ Not including patients who were referred for CTCA but diverted to other imaging modalities remains a major weakness of the study.

Notwithstanding these weaknesses, we believe that the methodology developed in our HASTE (Heart and Stroke Trust Endeavour) Standardised Operational Procedure may serve as a useful starting point in assisting other DGHs, particularly who do not have a PCI service, in developing a diagnostic CCT service particularly with deference to the 2016 NICE guidance update. ${ }^{15}$

Contributors ITP analysed the data and drafted the manuscript. CB analysed the data and reviewed the manuscript. JB assisted with the technological aspects and reviewed the manuscript. $\mathrm{AH}, \mathrm{EW}, \mathrm{MI}$ and $\mathrm{MH}$ reviewed the manuscript. EL contributed to overarching review of the content.

Funding The authors are grateful to the Heart and Stroke Trust Endeavour (HASTE), Registered UK Company Number 05185057, for their generous grant to publish this paper open access.

Competing interests None declared.

Patient consent Not required.

Provenance and peer review Not commissioned; externally peer reviewed.

Data sharing statement № additional data are available.

Open access This is an open access article distributed in accordance with the Creative Commons Attribution Non Commercial (CC BY-NC 4.0) license, which permits others to distribute, remix, adapt, build upon this work non-commercially, and license their derivative works on different terms, provided the original work is properly cited, appropriate credit is given, any changes made indicated, and the use is non-commercial. See:http://creativecommons.org/licenses/by-nc/4.0/.

\section{REFERENCES}

1. Bhatnagar P, Wickramasinghe K, Williams J, et al. The epidemiology of cardiovascular disease in the UK 2014. Heart 2015;101:1182-9.

2. Brennan JM, Redberg RF, Anderson H. V, et al. Low diagnostic yield of elective coronary angiography. 2010.

3. Meijboom WB, van Mieghem CA, Mollet NR, et al. 64-slice computed tomography coronary angiography in patients with high, intermediate, or low pretest probability of significant coronary artery disease. J Am Coll Cardiol 2007;50:1469-75.

4. Budoff MJ, et al. Diagnostic performance of 64-multidetector row coronary computed tomographic angiography for evaluation of coronary artery stenosis in individuals without known coronary artery disease results from the prospective multicenter ACCURACY (Assessment by Coronary Computed Tomographic Angiography of Individuals Undergoing Invasive Coronary Angiography) trial. J Am Coll Cardiol 2017;52:1724-32.

5. Miller JM, Rochitte CE, Dewey M, et al. Diagnostic performance of coronary angiography by 64-row CT. N Engl J Med Overseas Ed 2008;359:2324-36.

6. Douglas PS, Hoffmann U, Patel MR, et al. Outcomes of anatomical versus functional testing for coronary artery disease. N Engl J Med 2015;372:1291-300.
7. Scot-heart T. SCOT-HEART investigators. CT coronary angiography in patients with suspected angina due to coronary heart disease (SCOT-HEART): an open-label, parallel-group, multicentre trial. Lancet 2015;385:2383-91.

8. Foy $\mathrm{AJ}$, et al. Coronary computed tomography angiography vs functional stress testing for patients with suspected coronary artery disease: a systematic review and meta-analysis. JAMA Intern Med. 2017:1623-31.

9. Nielsen LH, et al. The diagnostic accuracy and outcomes after coronary computed tomography angiography vs. conventional functional testing in patients with stable angina pectoris: a systematic review and meta-analysis. Eur Heart J Cardiovasc Imaging. 2014:961-71.

10. Jørgensen ME, Andersson C, Nørgaard BL, et al. Functional testing or coronary computed tomography angiography in patients with stable coronary artery disease. J Am Coll Cardiol 2017;69:1761-70.

11. Puchner SB, Liu T, Mayrhofer T, et al. High-risk plaque detected on coronary CT angiography predicts acute coronary syndromes independent of significant stenosis in acute chest pain: results from the ROMICAT-II trial. J Am Coll Cardiol 2014;64:684-92.

12. Chow BJW, et al. HHS Public Access. 2016;35:981-9.

13. Deseive $S$, et al. Prognostic value of coronary computed tomography angiography during 5 years of follow-up in patients with suspected coronary artery disease. 2013:3277-85.

14. Moss AJ, Williams MC, Newby DE, et al. The updated NICE guidelines: cardiac CT as the first-line test for coronary artery disease. Curr Cardiovasc Imaging Rep 2017;10.

15. National Institute for Health and Care Excellence. NICE Guideline: Chest pain of recent onset : assessment and diagnosis. nice.org.uk/ guidance/cg95 (2016 update). 2016.

16. Abbara S, Blanke P, Maroules CD, et al. SCCT guidelines for the performance and acquisition of coronary computed tomographic angiography: a report of the society of cardiovascular computed tomography guidelines committee: Endorsed by the North American Society for Cardiovascular Imaging (NASCl). J Cardiovasc Comput Tomogr 2016;10:435-49.

17. Royal Colege of Radiologists, Royal College of Physicians, B. S. of C. I. BFCR(14)16. Standards of practice of computed tomography coronary angiography (CTCA) in adult patients. 2014.

18. Lempert $\mathrm{T}$, Brandt $\mathrm{T}$, Dieterich $\mathrm{M}$, et al. Original communications. 1991:140-6.

19. Agatston AS, Janowitz WR, Hildner FJ, et al. Quantification of coronary artery calcium using ultrafast computed tomography. J Am Coll Cardiol 1990;15:827-32.

20. Takx RA, Suchá D, Park J, et al. Sublingual Nitroglycerin Administration in Coronary Computed Tomography Angiography: a Systematic Review. Eur Radiol 2015;25:3536-42.

21. Gerber TC, Carr JJ, Arai AE, et al. lonizing radiation in cardiac imaging: a science advisory from the American Heart Association Committee on Cardiac Imaging of the Council on Clinical Cardiology and Committee on Cardiovascular Imaging and Intervention of the Council on Cardiovascular Radiology and Intervention. Circulation 2009;119:1056-65.

22. Qin J, Liu LY, Fang Y, et al. 320-detector CT coronary angiography with prospective and retrospective electrocardiogram gating in a single heartbeat: comparison of image quality and radiation dose. $\mathrm{Br}$ J Radiol 2012;85:945-51.

23. Maruyama T, Takada M, Hasuike T, et al. Radiation dose reduction and coronary assessability of prospective electrocardiogram-gated computed tomography coronary angiography: comparison with retrospective electrocardiogram-gated helical scan. J Am Coll Cardiol 2008;52:1450-5.

24. Castellano IA, et al. Journal of cardiovascular computed tomography a prospective national survey of coronary $\mathrm{CT}$ angiography radiation doses in the United Kingdom. J. Cardiovasc. Comput. Tomogr 2017;11:268-73.

25. System DCT, et al. Closing in on the K Edge : second- versus a thirdgeneration. 2014;273:373-82.

26. Apfaltrer $\mathrm{G}$, Szolar $\mathrm{DH}$, Wurzinger $\mathrm{E}$, et al. Impact on image quality and radiation dose of third-generation dual-source computed tomography of the coronary arteries. Am J Cardiol 2017;119:1156-61.

27. Pavitt CW, Harron K, Lindsay AC, et al. Deriving coronary artery calcium scores from CT coronary angiography: a proposed algorithm for evaluating stable chest pain. Int $J$ Cardiovasc Imaging 2014;30:1135-43. 